Laparotomy was decided on. At 10 p. m. Dr. Joseph W. McGill operated under ether anesthesia. A 4-inch incision was made outside of the right rectus muscle, liver or intestinal injury as well as perirenal trouble being suspected. liree blood, containing many large clots, was present in the abdominal cavity. Examination of the bladder, intestines and liver disclosed nothing. The posterior peritoneum of the right side was raised by the pressure of the blood and was loosely applied to the parietes. In the region of the cecum the posterior peritoneum was bulging over a large hematoma. Above this point there was a tear in the peritoneum.

After palpation of both kidneys, and swabbing out the major portion of the blood from the general abdominal cavity, the peritoneum of the right posterior wall was stitched in place and the abdomen closed, one drain being directed through a stab incision in the right lower quadrant to the retroperitoneal area, and the peritoneum sutured around it. The pelvis also was drained.

After operation the temperature rose slightly, reaching 102.6 on the following day. The urinary findings were still negative. On the sixth day after operation, after both drain tubes had been withdrawn, the pathologic report on the urine indicated some microscopic blood present in the sample. Macroscopic blood was noted the following day. The amount of hloorl increased daily until, on the tenth day after operation. the urinary specimen showed a large proportion of blood. The blood content then gradually decreased until the specimen was reported as negative on the twenty-second day: Hexamethylenamin was given in 3-grain doses on the first day of urinary signs, and was continued until the report was returned negative. The hematuria was not considered to warrant operation, as it was assumed that the contusion or laceration would heal spontaneously. A subsequent true nephritis was not considered probahle in the prognosis.

The child coughed a little on entrance to the hospital, and the suggestion was marle that this might be attributed to cliaphragmatic irritation. This cough disappeared without special treatment during the stay in the hospital. The child sat up in a chair on the twenty-fourth day; was pushing herself around the ward in a whecl chair on the twenty-sixth day, and was up and around on the twenty-eighth day after operation. Since discharge the child has been doing well and no complications lave been reported.

AC'LTE POISONING; BY FIVE GRATNS OF ACETYLSAIIVYIIC ACID-_ASPIRIN"

EDMend P. Shelay, M.D.. New Jiak

Attending Physician, (ity llospital

The following report shows that some persons are peculiarly susceptible to the effect of acetylsalicylic acid"aspirin":

Miss A., a strong, vigorous woman, aged 32 , weighing 140 pounds, consulted me about indefinite pains in the back and chest which followed a slight sore throat. I prescribed aspirin in 5-grain capsules. The first capsule, of the Bayer brand of acetylsalicylic acid, was taken at $12: 15$. Forty-five minutes later the patient felt an itching of the scalp, swelling of the hands, and whitc blotches appeared over the face and body. In a few minutes the eyes were swollen and closed and violent irritation of the larynx set in.

I was hastily summoned and found the patient unable to speak and with marked interference with breathing. The face was hardly recognizable: and the obstruction of the larynx was very alarming. The pulse was rapid and the breathing spasmodic. The question of tracheotomy for the relief of the laryngeal obstruction was considered.

Before my arrival the patient had taken half an ounce of Epsom salt. This soon brought on nausea and a smalt amount of liquid was vomited, after which the patient fainted. On her return to consciousness the rash began to fadc, the breathing became less labored, and the swelling of the eyes subsided. By 3 o'clock, two hours and forty minutes from the time she had taken the acetylsalicylic acid, the patient was fairly comfortable, could open her eyes and speak. At that lime the temperature was 98.6. respiration 20. pulse 92
A rapid return to normal followed. The ne:a ray no ill effects were apparent.

That these symptoms were caused by the acetylsalicylic acid there can be no doubt, as the patient had not partaken of food or of other medicine since the night before. Unusual susceptibility to the action of salicylates has often been noted, but I do not recall a case in which so small a dose has produced such marked symptoms.

116 West Seventy-Fourth Street.

\section{AN ASPIRATOR FOR PARACENTESIS}

$$
\text { H. C. Stevens, M.D., Dunning, Ill. }
$$

Many forms of apparatus have been devised to create a vacuum sufficient to withdraw fluid from the pleural cavities. The Pétain system is effective when the parts are new. It has the disadvantage characteristic of all complicated mechanisms of easily getting out of order. The two chicf difficulties, aside from expense, which we have experienced have been (1) in the valves of the pump, which have failed to close properly, and (2) in leakage about the hard rubber stopper of the bottle. The latter trouble is probably due to changes in the rubber incident to age. To obviate these difficulties, we have devised a simple apparatus which produces suction by means of a siphon. Any hospital possessing two 2-liter bottles, two two-way rubber stoppers, some glass tubing and rubber tubing can assemble an effective aspirator in a very short time. The construction is shown in the drawing. A 2-liter bottle, provided with a two-way rubber stopper, is filled with water and serves as the siphon. In the illustratim. I), with the tube, lE. performs this function. A similar bottle, $B$, is sterilized by boiling, together with the tuties .1 and $C$. Bottle $B$ is used to collect the effusion. Rubber tubing with at inside diametor of $4 \mathrm{~mm}$. is used. A blunt trocar with a sharp obturator, or a smaller steel needle may be used. A "window" consisting of $2 \mathrm{~cm}$. of glass tubing is inserted into tubc $A$, a short distance from the necile. Tube $E$ is filled with water and clamped, or allowed to kink from its own weight, at the point where it is connected with the glass tube. If the latter method is followed, simply raising tube $E$ will cause the siphon to run; lowering it will stop it. In performing paracentesis, $A, B, C$ and the necdlo are sterilized by boiling. The siphon is started aisd then stopped. Puncture is made in the manner, and at the sitc. preferred by the operator. An assistant starts the siphon as soon as the needle enters the pleural cavity.

Chicago State Hospital.

The Human Thought Mechanism.-The intellect cannot examine any fact of matter or energy directly. The telephoilc mechanism of the senses is always in between. Like the line officer in the war. we live in a dugout. the dark dugout of the cranial cavity. Our news comes in over the wires or afferent nerves. Our news is never complete, for our sensc organs are not adapted to all the forces of nature. Our news is often faulty because the sense organs or the transmission is imperfect. Often we fail to get messages becausc our receiving operators are indolent, or doing other things. Moreover, if we get the news straight, we may not use it right; that is, make the right conclusion or judgment.-E. P. Iyon, Tiras State Journal of Mcdicine. 